BMC

Plant Biology

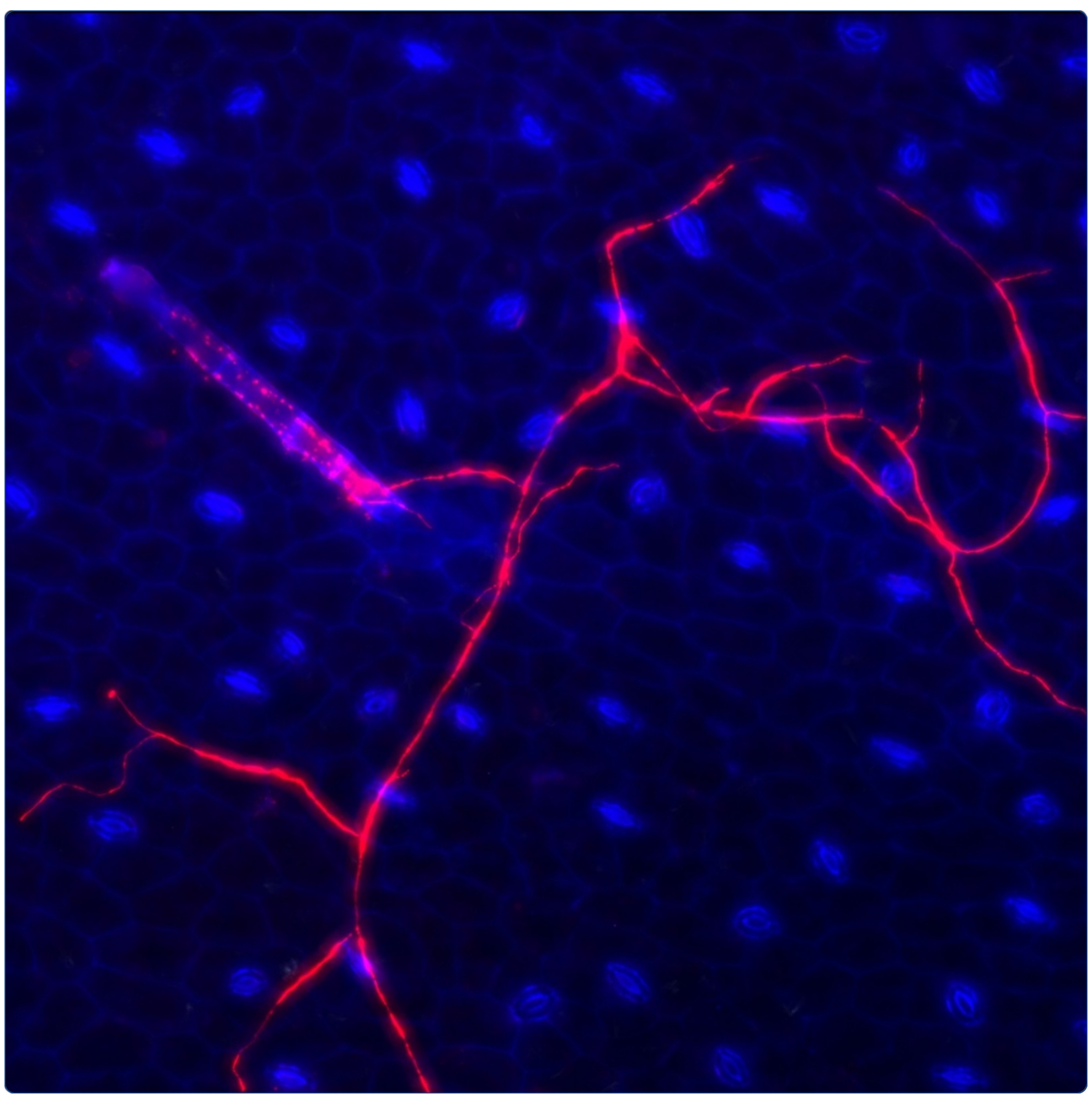

CbCTB2, an O-methyltransferase is essential for biosynthesis of the phytotoxin cercosporin and infection of sugar beet by Cercospora beticola

Staerkel et al.

C BioMed Central 


\title{
CbCTB2, an O-methyltransferase is essential for biosynthesis of the phytotoxin cercosporin and infection of sugar beet by Cercospora beticola
}

\author{
Cornelia Staerkel ${ }^{1}$, Marike J Boenisch ${ }^{1}$, Cathrin Kröger ${ }^{1}$, Jörg Bormann ${ }^{1}$, Wilhelm Schäfer ${ }^{1}$ and Dietmar Stah $2^{*}$
}

\begin{abstract}
Background: Cercospora leaf spot disease, caused by the fungus Cercospora beticola, is the most destructive foliar disease of sugar beets (Beta vulgaris) worldwide. Cercosporin, a light-inducible toxin, is essential for necrosis of the leaf tissue and development of the typical leaf spots on sugar beet leaves.

Results: In this study we show that the O-methyltransferase gene CTB2 is essential for cercosporin production and pathogenicity in two C. beticola isolates. We established a transformation system for C. beticola protoplasts, disrupted CTB2, and transformed the $\Delta c t b 2$ strains as well as a wild type strain with the DsRed reporter gene. The $\Delta c t b 2$ strains had lost their pigmentation and toxin measurements demonstrated that the $\Delta c t b 2$ strains were defective in cercosporin production. Infection of sugar beets with the wild type and $\Delta$ ctb2 DsRed strains showed that the deletion strain was severely impaired in plant infection. Histological analysis revealed that the CTB2deficient isolate cannot enter the leaf tissue through stomata like the wild type.

Conclusions: Taken together, these observations indicate that cercosporin has a dual function in sugar beet infection: in addition to the well-known role in tissue necrosis, the toxin is required for the early phase of sugar beet infection.
\end{abstract}

\section{Background}

Cercospora beticola, an ascomycete, is the economically most important foliar pathogen of sugar beet (Beta vulgaris). Characteristic symptoms of $C$. beticola infection include brown leaf spots extending from the adaxial to the abaxial sides of the leaf; from spring to summer the spots increase in number as the disease progresses, finally coalescing such that the entire leaf turns brown and shrinks. Leaf spot disease is economically critical because the destruction of the leaves limits photosynthesis and compromises yield [1].

C. beticola propagates by macroconidia, asexual spores that overwinter in plant debris on the field and are spread in spring by wind and rain splash [2]. C. beticola is a hemibiotrophic fungus with biotrophic and necrotrophic phases. Approximately 3-4 days after inoculation, the fungus enters the host through open stomata [3]; after penetration, fungal hyphae grow intercellularly and colonize

\footnotetext{
* Correspondence: dietmar.stahl@kws.com

${ }^{2}$ KWS SAAT AG, Grimsehlstr. 31, Einbeck 37555, Germany

Full list of author information is available at the end of the article
}

the leaf parenchymal tissue in an asymptomatic manner $[4,5]$. The final, necrotrophic phase is characterized by the formation of necrotic lesions, resulting in the leaf spots that are the typical sign of this disease. Unlike other leaf pathogens that necrotize from pinpoint lesions and expand outward, lesions produced by $C$. beticola involve the near-simultaneous collapse of cells in an area many millimeters in diameter. The necrotized tissue becomes the site of conidiophore and conidial development. Although lesions can expand after the initial tissue collapse, the increase in necrotic area on the leaf surface is due primarily to an increase in the number of lesions. One sporulation cycle under field conditions lasts approximately 12 days [1].

The Cercospora family has many members that have different hosts, including tobacco, soybean, coffee, rice, corn, and peanut [6]. Cercospora species produce cercosporin, a photo-activated toxin that generates reactive oxygen species upon exposure to light. The

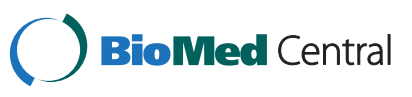

(C) 2013 Staerkel et al.; licensee BioMed Central Ltd. This is an Open Access article distributed under the terms of the Creative Commons Attribution License (http://creativecommons.org/licenses/by/2.0), which permits unrestricted use, distribution, and reproduction in any medium, provided the original work is properly cited. 
cercosporin biosynthesis pathway in $C$. nicotianae has been shown to include eight genes, CTB1-CTB8. CTB1, a polyketide synthase, condenses and decarboxylates the precursors malonyl-CoA and acetyl-CoA to form a polyketide. It then catalyzes Claisen condensation and ring closure of the molecule. The next steps are oxidation and hydration reactions executed by $C T B 3$, an $\mathrm{O}$-methyltransferase and FAD-dependent monooxygenase, CTB5, a FAD/FMNdependent oxidoreductase, CTB6, a NADPH- dependent oxidoreductase, and CTB7, another FAD/FMN-dependent oxidoreductase. The subsequent methylation step is carried out by CTB2, also an O-methyltransferase, and CTB3. The resulting polyketomethylene is dimerized and then exported from the cells by CTB4, a major facilitator superfamily transporter. This export mechanism leads to autoimmunity of $C$. beticola against its own toxin. Cercosporin expression is regulated by the zinc-finger transcription factor CTB8.

Previous studies have demonstrated that individual inactivation of CTB1, CTB2, CTB3, or CTB8 leads to the transcriptional inhibition of the entire gene cluster and prevents cercosporin production in $C$. nicotianae $[7,8]$. The current study aimed to determine whether cercosporin production in C. beticola could be reduced by a single knockout mutation, and to identify differences in the infection behaviors of wild type and toxin-deficient strains.

\section{Results}

Disruption of CTB2 in the fungal isolates Ahlburg and Ferrara

Primers were designed using the homologous sequence from C. nicotianae (accession number DQ991505). CTB2 of isolate Ferrara was amplified via PCR; sequencing revealed that the coding region of the $C b C T B 2$ gene is $91.6 \%$ identical to the coding region of $C n C T B 2$ gene. The derived amino acid sequence of $C b C T B 2$ is $96 \%$ identical to the protein from $C$. nicotianae.

The gene was disrupted in two isolates of C. beticola, Ferrara and Ahlburg. Transformation resulted in 44 Ferrara and 23 Ahlburg transformants. For both isolates, two independent transformants were selected with a disrupted CTB2 gene (Figure 1). In the Ferrara isolate, $C T B 2$ gene disruptions $(\Delta c t b 2)$ are subsequently called $F \Delta c t b 2-1$ and $F \Delta c t b 2-2$ and accordingly in the Ahlburg isolate $A \Delta c t b 2-1$ and $A \Delta c t b 2-2$. Ectopic transformants from each wild type were selected and called Fec (Ferrara) and Aec (Ahlburg).

The Ahlburg wild type strain and the deletion mutants $A \Delta c t b 2-1$ were selected for transformation with DsRed resulting in more than 20 DsRed fluorescent transformants of each strain. DsRed reporter strains were obtained by transformation of C. beticola with pII99DsRed [9], which was linearized with XhoI. CBT2 deletion mutants

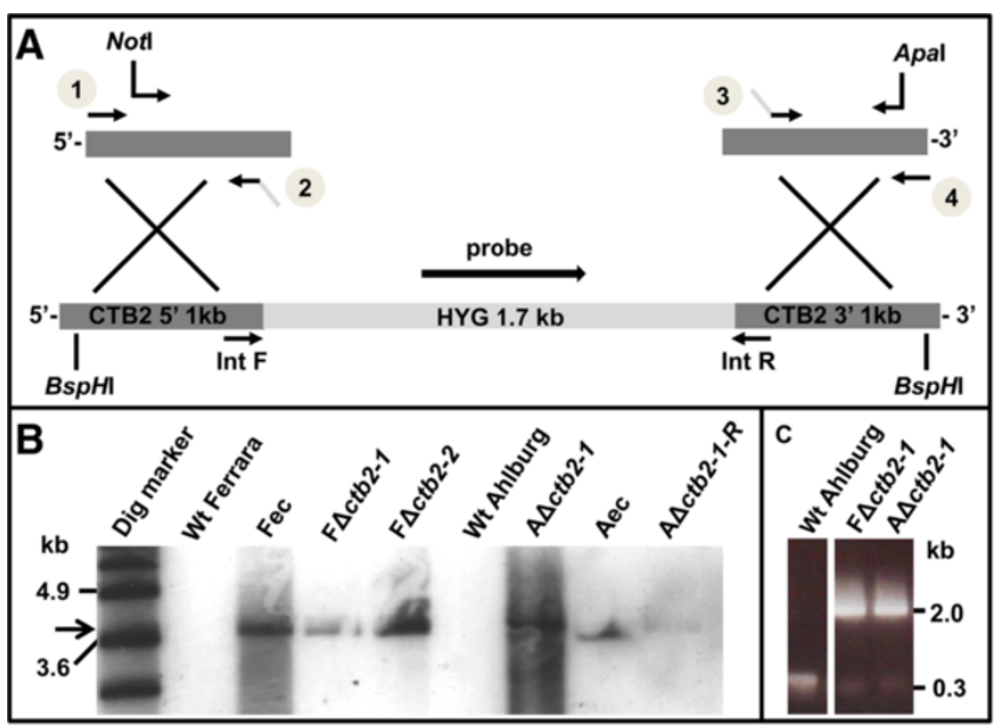

Figure 1 Disruption of CTB2 gene in C. beticola isolates Ahlburg and Ferrara. A The $800 \mathrm{bp} 5^{\prime}$ and $3^{\prime}$ fragments of CTB2 were amplified separately with primer pairs $1 / 2$ and $3 / 4$, fused with the hygromycin cassette by overlapping regions, and amplified with nested primers (including Notl and Apal sites) before cloning into pGEMT. B Example of Southern blotting with a hygromycin probe, from left to right, wild type Ferrara (no band), Ferrara ectopic, Ferrara ctb2 gene disruption strains 2-1 and 2-2, wild type Ahlburg, Ahlburg ctb2 disruption strain 2-1, Ahlburg ectopic, Ahlburg ctb2 disruption strain 2-2 (band at 3.7 kb, black arrow). Dig VII marker (Roche) was used as DNA size ladder. C Confirmative PCR with CTB2 internal primers located adjacent to the integration locus (IntF and IntR), from left to right, wild type Ahlburg (band at $0.3 \mathrm{~kb}$ ), Ferrara cbt2 disruption strain 2-1, Ahlburg disruption strain 2-1 (band at $2 \mathrm{~kb}$ ) F $\mathrm{Actb2}-2$ and $A \Delta c t b 2-2$ produced the same PCR bands as the correspondent disruptants (data not shown). 
transformed with DsRed were pink because the red protein was visible in the white hyphae. A DsRed Ahlburg wild type strain expressing DsRed (wt Ahlburg-R) and $A \Delta c t b 2-1$ expressing DsRed $(A \Delta c t b 2-1-R)$ was chosen for further analysis. Growth rates and conidiation of the transformants were not changed.

\section{$\Delta c t b 2$ transformants are reduced in pigmentation and toxin production}

The gene disruption strains lost the characteristic dark pigmentation and appeared white, while the ectopic and wild type colonies were gray. Other properties such as conidiation and growth rate remained unchanged (data not shown).

The Ahlburg isolate had a higher toxin content than the Ferrara isolate (Figure 2), which may explain why Ahlburg is the more virulent isolate. The $\Delta c t b 2$ strains from Ahlburg and Ferrara isolates did not produce cercosporin, whereas the toxin content of the ectopic Ferrara transformant was identical to the wild type isolate (Figure 2). Ahlburg ectopic transformant produced similar amounts of cercosporin as the wild type (data not shown).

When in vitro-cultured sugar beets were treated with aqueous solution of cercosporin from PDA plates, the plants dipped in wild type extract died after one day, while plants treated with the water control or the $\Delta c t b 2$

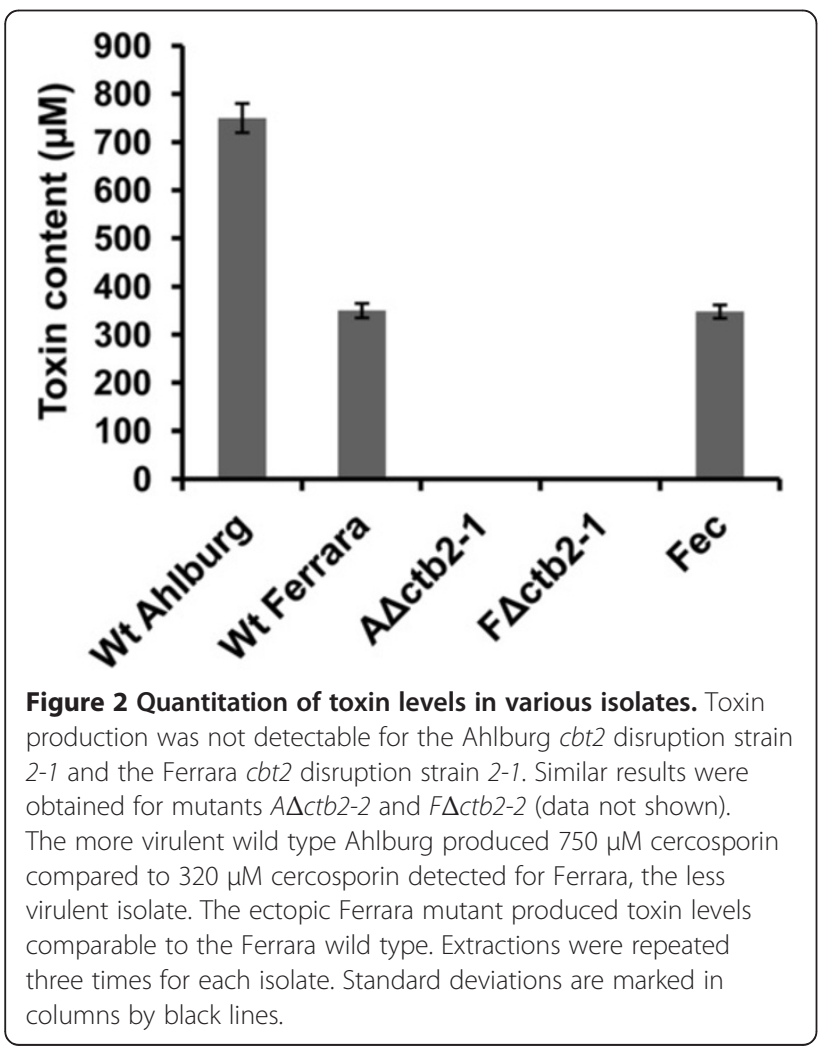

extract remained healthy and green (Figure 3). These experiments were repeated three times with five plants treated in each plate extract. Culture extract from the gene disruption strain therefore lacks a key component needed to damage plant tissue.

\section{CTB2 is important for pathogenicity of C. beticola}

Infection of 3-month-old sugar beets with the Ahlburg and Ferrara wild type and the corresponding disruptants $A \Delta c t b 2-1$ and $A \Delta c t b 2-2$ as well as $F \Delta c t b 2-1$ and $F \Delta c t b 2-$ 2 strains demonstrated that the $\Delta c t b 2$ strains of both isolates caused no lesions on the leaves, and the entire plants appeared healthy three weeks after infection. Symptoms caused by the two disruptants of each wild type were indistinguishable (data not shown). Wild type strains of Ahlburg and Ferrara infected the plants and caused lesions. The Ahlburg isolate proved to be highly aggressive causing severe leaf spot lesions comparing to the less virulent Ferrara isolate causing only minor lesions (Figure 4). The symptoms caused by the ectopic transformants of Ferrara and Ahlburg were indistinguishable from the symptoms of the corresponding wild type strain, indicating that CTB2 is essential for pathogenicity of C. beticola (Figure 4).

Disease rating was carried out by assessing the percentage of diseased leaf surface [10]. The disease rating 21 days after infection demonstrated that the plants infected with the gene disruption strain were symptomfree, while the plants infected with the Ahlburg wild type strain had a disease index of 4 on a scale of 9 , whereas Ferrara wild type and ectopic strains had a disease index of 1 (Figure 5).

Occasionally, single lesions were observed on some leaves infected with the Ahlburg $\Delta c t b 2$ strains 24 days after inoculation. The application of two different inoculation techniques showed that the appearance of the rare lesions depends on the quality of inoculum. A rough inoculum with clumps of mycelium, which was produced as described [11] favored the appearance of lesions. However, the preparation of a fine spore suspension by filtering and sieving, as described in Material and Methods section, resulted only in very few necrotic spots after 24 days.

\section{$\Delta c t b 2$ is impaired in penetration of host leaves}

A histological analysis was performed to evaluate the infection process of the Ahlburg wild type and the Ahlburg $\Delta c t b 2-1$ strain at the cellular level. A constitutive expression of the DsRed fluorescent reporter in Ahlburg wild type and Ahlburg disruptant facilitated the microscopic detection of fungal hyphae. C. beticola infection monitored by fluorescence microscopy showed a strong DsRed fluorescence in the necrotic spots caused by the DsRed wild type strain (Figure 6A, C). The 


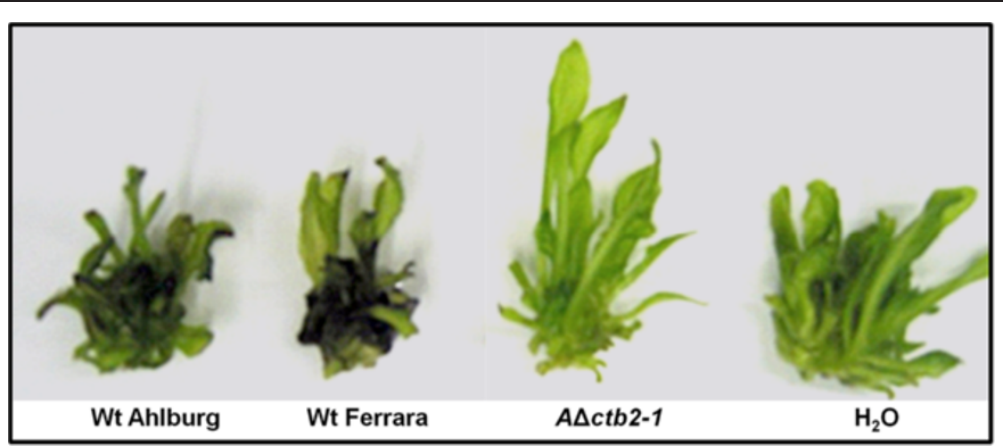

Figure 3 Sugar beet plants grown in-vitro and treated with toxin extracts. Plants exhibited varying degrees of damage one day after treatment with cercosporin extracts from various fungal isolates and mutants. Plants treated with cercosporin from wild type Ahlburg and Ferrara became necrotic and black (left), but plants treated with cercosporin extract of the Ahlburg disruption strain 2-1 remained green and healthy, comparable with the plant treatment with water which served as the negative control (right). Experiments were repeated three times with five plants for each extract.

$\Delta c t b 2-1$ DsRed strain did not cause leaf spots and the fluorescence was restricted to the hyphae on the leaf surface (Figure 6B, D).

Fluorescence microscopy of the adaxial and abaxial leaf surfaces showed germination and hyphal spread of the Ahlburg wild type (Figure 7A) and the gene disruption strain (Figure 7B). The wild type strain was beginning to infect through the stomata at 4 days after infection (Figure 7C) while the gene disruption strain did not penetrate the host tissue (Figure 7D). Cross sections of leaves at different stages of the infection revealed that the wild type had progressed intercellularly from the penetration site (Figure 8A) and was beginning to cause necrosis 14 days after infection. The gene disruption strain stopped at the epidermis layer (Figure $8 \mathrm{~B}$ ). The wild type spread massively inside the leaves until 21 days after infection (Figure 8C), when large necrotic lesions became visible on the surface. The gene disruption strain had ceased to grow at this point and failed to spread inside the leaf (Figure 8D).

\section{Discussion}

Despite progress in breeding and fungicide-mediated plant protection, understanding of the molecular interaction between C. beticola and sugar beet is just beginning. Previous studies in C. nicotianae indicated that the disruption of several genes involved in toxin biosynthesis disabled cercosporin production and rendered the fungus nonpathogenic [12]. In the current study, we showed that the O-methyltransferase gene CTB2 is essential for cercosporin biosynthesis and fungal virulence of $C$. beticola. Knock-out mutants (Figure 1) failed to produce cercosporin (Figure 2) and were nonpathogenic on sugar beet plants grown in the greenhouse or in vitro. The mutant strains lost the characteristic dark pigmentation.

The loss of grey pigmentation of the $\Delta c t b 2$ strains indicates that the disturbance of the cercosporin pathway may affect the formation of fungal pigments. Mycelial pigments like melanin or bikaverin and fusarubins of Fusarium fujikuroi $[13,14]$ are synthesized by polyketide synthase gene clusters similar to the Cercosporin

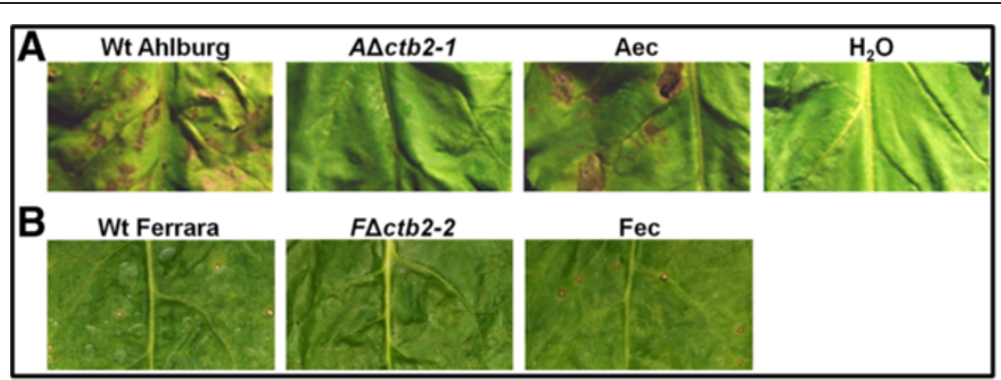

Figure 4 The $\triangle C T B 2$ strains fail to cause disease symptoms on sugar beet leaves. A Enlarged sections of the surface of sugar beet leaves reveal that exposure to the wild type Ahlburg strain led to the characteristic leaf spot symptoms 21 days after inoculation. Ahlburg ctb2 disruption strain 2-1 failed to cause typical leaf spot symptoms. Ahlburg ectopic strain produced wild type like leaf spots. Treatment with water served as the negative control. B Ferrara wild type caused leaf spot symptoms, although significantly smaller compared to Ahlburg wild type. Ferrara cbt2 disruption strain 2-1 failed to cause leaf spot symptoms in contrast to Ferrara ectopic mutant. Experiments were repeated twice with ten plants for each fungal strain. Both disruptants of each wild type isolate gave similar results (data not shown). 


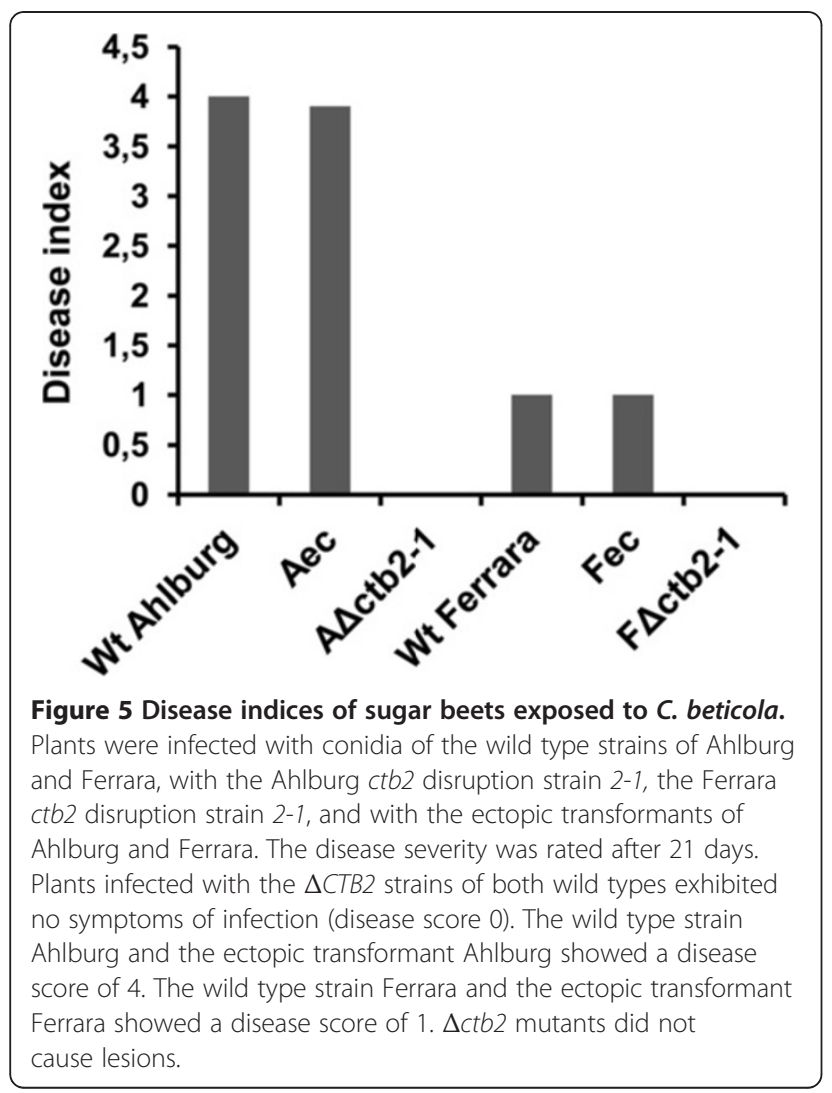

biosynthesis gene cluster. Analysis of $\Delta c t b 2$ mutants of $C$. nicotianea revealed that also transcripts of CnCTB1, CnCTB3, CnCTB4 and CnCTB8 are completely down regulated when $C n C T B 2$ was disrupted [7]. Beside the involvement in cercosporin biosynthesis the CbCTB2 protein or proteins targeted by a CbCTB2 feedback inhibition could be involved in the biosynthesis of other fungal pigments. Beside the essential role for cercosporin biosynthesis the knock-out of the O-methyltransferase gene CTB2 may therefore have a pleiotropic effect on C. beticola. A discoloration of the fungal mycelium was also described for the $\Delta c t b 1$ and $\Delta c t b 3$ mutants of $C$. nicotianae and explained by the absence of the red-colored cercosporin [15].

Weiland et al. [16] performed site-directed knock-out experiments of $C b C T B 1$ in $C$. beticola, the polyketide synthase involved in cercosporin biosynthesis. Two analyzed $\Delta c t b 1$ mutants did not produce cercosporin, yet the mutants were able to cause smaller and fewer lesions than the wild type. We observed that the $\Delta c t b 2$ strains also caused rarely necrotic spots, but the frequency of this spots was influenced by the quality of the inoculum. The more the infection technique corresponded to the natural infection condition; the lower was the number of necrosis.

Plant pathogenic fungi must overcome the cell wall in order to enter the host plant, and have therefore developed very efficient and sophisticated mechanisms to
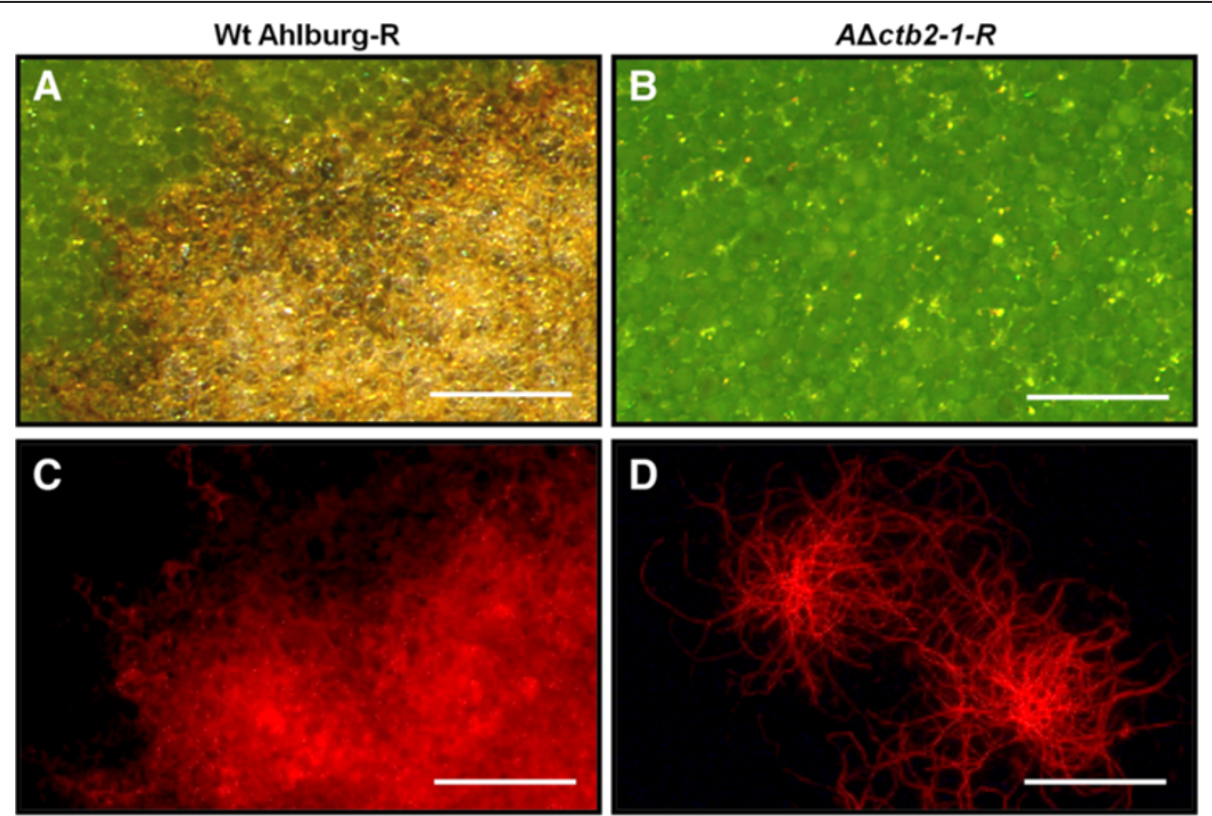

Figure 6 C. beticola infection monitored by fluorescence microscopy. Imaging leaf infection of sugar beet plants with the wild type Ahlburg and the Ahlburg ctb2 disruption strain 2-1 both transformed to express constitutively DsRed. A and B Bright field images of the leaf surface, demonstrate characteristic leaf spot symptoms of the wild type infection at 21 days after inoculation, while the gene disruption strain did not cause leaf spots. $\mathbf{C}$ and $\mathbf{D}$ The mycelium of DsRed expressing strains of Ahlburg wild type and Ahlburg ctb2 disruption strain 2-1 growing on the leaf surface was detected with DsRed filterset of Leica MZ FL III microscope. Scale bars: $250 \mu \mathrm{m}$. 

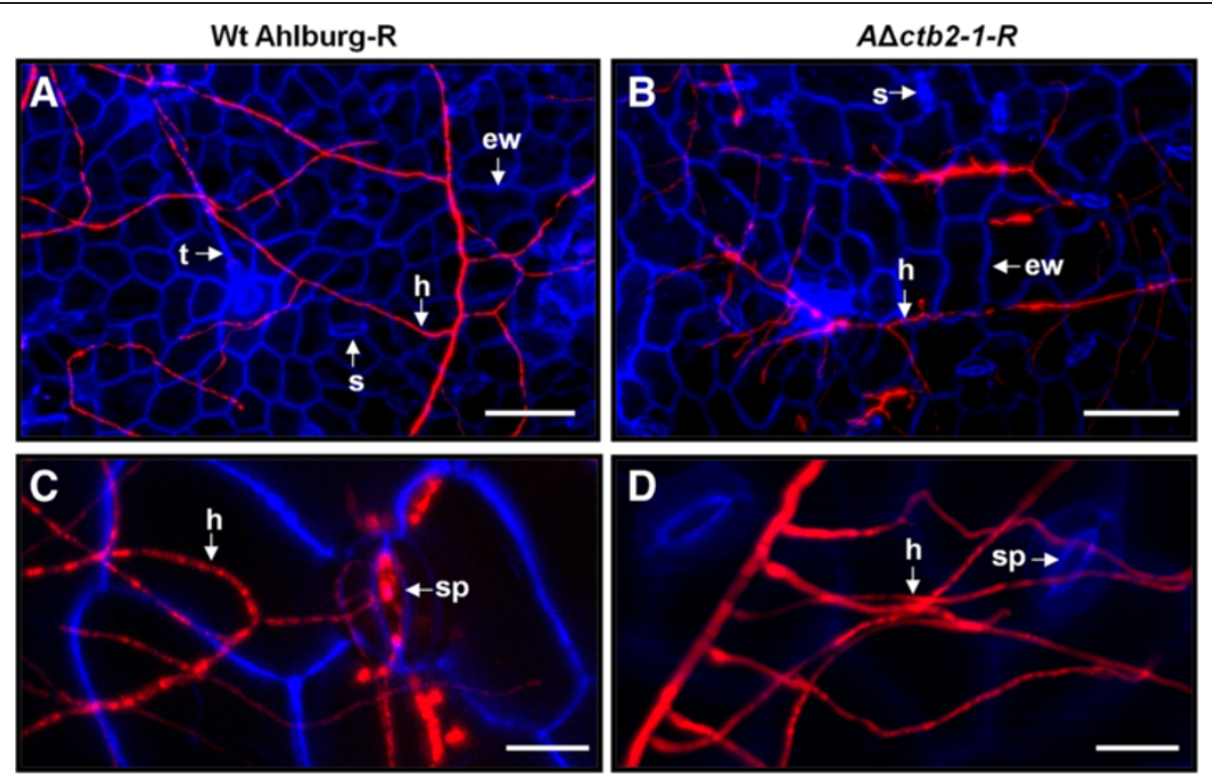

Figure 7 Colonization and penetration of sugar beet leaves by Ahlburg wild type DsRed and Ahlburg ctb2 disruption strain 2-1-DsRed. Fluorescence microscopy was performed with a Zeiss fluorescence microscope, using the DsRed filter for detection of the hyphae and UV light to excite blue autofluorescence of the plant cell wall. At 4 days after inoculation, A Ahlburg wild type-DsRed mycelia and B Ahlburg ctb2 disruption strain 2-1-DsRed mycelia were visible on the surface of leaves. C At 4 days after inoculation, the wild type approached stomata and began to penetrate. D The gene disruption strain grew on the surface without entering through stomata at 4 days after infection. Scale bars: $\mathbf{A}$ and $\mathbf{B}=100 \mu \mathrm{m}, \mathbf{C}$ and D $=20 \mu \mathrm{m}$. Abbreviations: ew epidermal cell wall, $\mathrm{h}$ hyphae, s stomata, sp stomatal pore, $\mathrm{t}$ trichome.

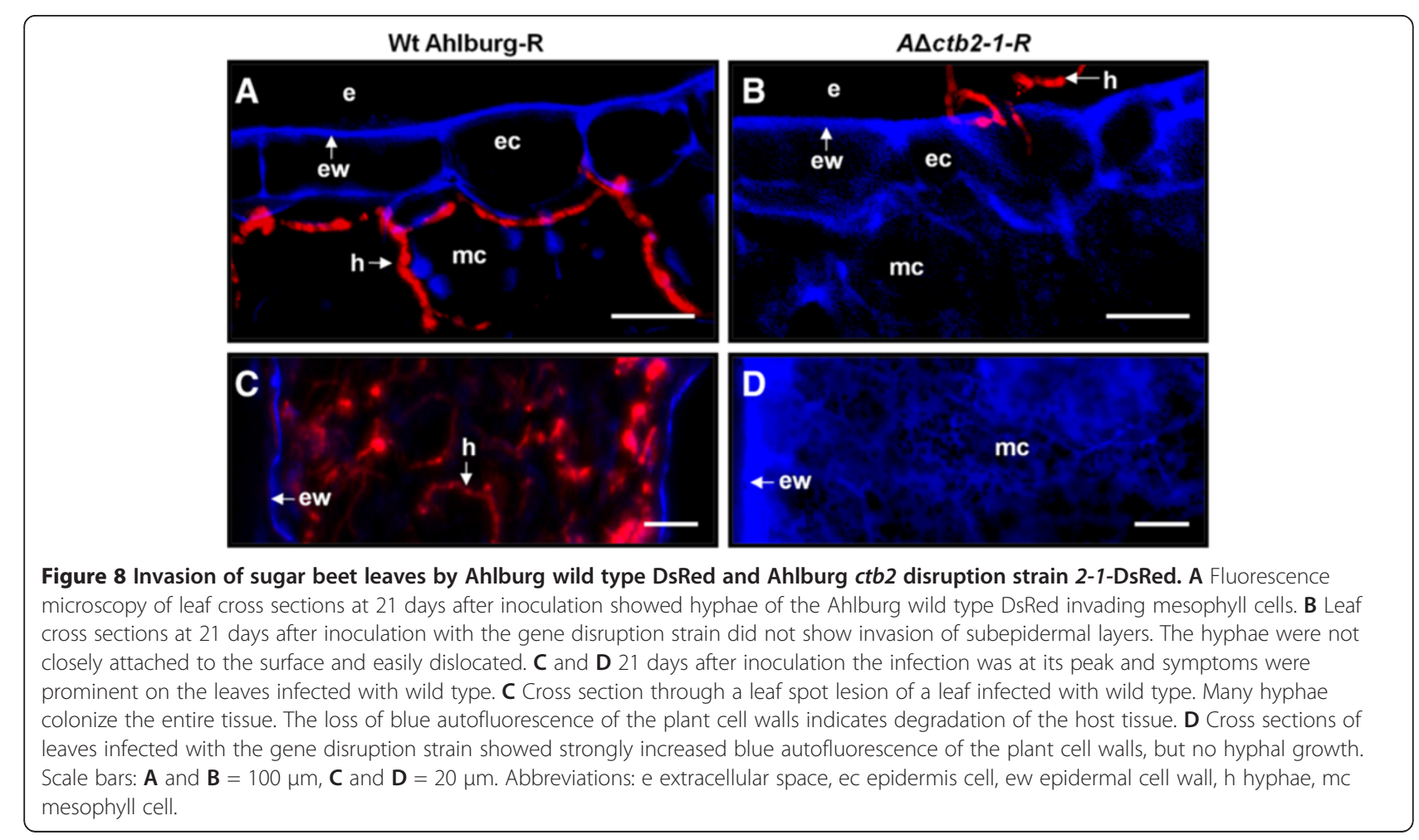


breach that barrier. The mode of fungal penetration depends on their lifestyle; $C$. beticola has been shown to enter the host leaves without specific infection structures, invading through the stomata, followed by intercellular growth [4].

Our observations via fluorescence microscopy of reporter strains revealed that the Ahlburg $\Delta c t b 2$ strain is unable to penetrate host tissue and establish an infection. Although the mutant strain germinated well on the surface, maintained growth for several days, and in some cases was able to localize stomata, it failed to grow inside the leaves or to cause disease symptoms, indicating that cercosporin is necessary for tissue colonization during early stages of infection by $C$. beticola. The involvement of cercosporin in the early infection phase of $C$. beticola is unexpected. Earlier studies revealed that purified cercosporin caused necrotic spots on sugar beet leaves that were similar in size and ultrastructural composition to the lesions caused by $C$. beticola [5,17]. Therefore, it was hypothesized that cercosporin's major contribution was to cause the extensive blighting symptoms characteristic of many Cercospora diseases. After penetration through the stomata and colonization, the membrane-damaging toxin should allow cell breakdown and leakage of nutrients for fungal growth and sporulation [6].

The failure of the CTB2-deficient strains to penetrate and colonize leaf tissue raises questions regarding the role of cercosporin in the biotrophic phase of infection. Transcriptome analysis of more than 17.000 sugar beet cDNAs demonstrated that the transcriptional activation of defense reactions in the early to mid stages of $C$. beticola infection is suppressed in polygenic resistant or susceptible genotypes [1]. C. beticola has obviously developed a strategy to dampen the immunity triggered by pathogen-associated molecular patterns (PAMP). In the case of the C. beticola-sugar beet interaction, the suppression of phenylalanine-ammonium lyase and cinnamate4-hydroxylase have been described in detail [11]. The repression of phenylalanine-ammonium lyase gene expression was induced by $C$. beticola production of phytohormone abscisic acid during the early phase of infection [18].

It is interesting to speculate that cercosporin may be involved in the suppression of plant defense reactions. As a prerequisite for this model, the toxin must be present in the early phase of infection and easily accessible to the plant cells. Since the biosynthesis of cercosporin is light-regulated [19], it can be assumed that the germinating spores and growing hyphae of $C$. beticola are already producing the toxin on the surface of the epidermis. Furthermore, cercosporin is a lipidsoluble molecule that penetrates rapidly into host cell membranes [6] and can interact with membraneembedded molecules such as receptors or transporters. However, additional experiments are necessary to demonstrate a direct role of cercosporin in the repression of plant defense mechanisms.

\section{Conclusions}

We disrupted the CbCTB2 gene coding for an Omethyltransferase thereby eliminating toxin production. The resultant mutants were not only unable to produce the toxin, but failed to infect sugar beet leaves. Cercosporin deficient mutants grow on the leaf surface but were unable to invade and colonize sugar beet leaves. Previously, it was hypothesized that cercosporin is involved in tissue damage after initial penetration and colonization. Here we show that cercosporin plays also a role in the initial biotrophic phase of the pathogen. Recent results showed that Cercospora beticola represses transcriptional activation of plant defense pathways. We assume that cercosporin is important for suppressing these defense reactions which are otherwise triggered by pathogen associated molecular patterns. Thereby, cercosporin enables the pathogen to successfully infect its host plant.

\section{Methods}

\section{Primers and plasmids}

Amplification of the hygromycin cassette required primers YgF (5'-GTTGGCGACCTCGTATTGG) and HyR (3'-CT TACCACCTGCTCATCACCT). The primers CTB2-1F (5'-CGCTAGATTTAGGTGTTGGA), CTB2-2R (5'-agatg ccgaccgaacaagagctgtccccGCAATCTTTCTTCCTATG CT), CTB2-3F (5'-caatgctacatcacccacctcgctcccccGTT TCCAAGTCCAAGATCTG), CTB2-4R (5'-CTCTTTCG TCCCTCGTATCTC), CTB2-5F (5'-AACCTCCTTTGCG TATTCTC), and CTB2-6R (5'-ATGTTTCCGAGTTCTT GATGTG) were used for the CTB2 deletion (hygromycinoverlapping sequences underlined). Finally, primers CTB2-int-F (5'-AGCATAGGAAGAAAGATTGC) and CTB2-int-R (5'-CAGATCTTGGACTTGGAAACG) served as control primers for the CTB2 gene. Primers were designed using the homologous sequence from $C$. nicotianae (accession number DQ991505). Plasmids used in this study were pGEMT (Promega, Mannheim, Germany) for TA cloning of PCR fragments, pII99DsRed [9], and pGEMThyg (Le Thi Thu Giang, University of Hamburg, Germany).

\section{Fungal and bacterial strains}

Escherichia coli strain XL1-blue (Stratagene, La Jolla, CA, USA) was used for molecular cloning. The $C$. beticola isolates Ahlburg and Ferrara were kindly provided by Planta GmbH, Einbeck, Germany. Ahlburg is known to be the more virulent isolate (unpublished results). 


\section{Media}

C. beticola was cultured in complete liquid media [20] for DNA extraction, and on complete media agar plates for maintenance. Transformants were grown under selective pressure with $50 \mu \mathrm{g} / \mathrm{ml}$ hygromycin B (Duchefa, Netherlands) and $100 \mu \mathrm{g} / \mathrm{ml}$ geneticin (Invitrogen, Germany), respectively. Toxin production was carried out on potato dextrose agar (PDA) plates (pH 5.6) under ambient light. Bacteria were cultured in Luria-Bertani liquid media or on Luria-Bertani plates with $200 \mathrm{mg} / \mathrm{ml}$ ampicillin (Sigma Aldrich, Munich, Germany). MS medium for the in vitro culture of sugar beets was prepared as described [21].

\section{Fusion PCR}

CTB2 was disrupted by double homologous recombination using fusion PCR to generate a construct consisting of a 1219-bp $3^{\prime}$ part of the gene, the hygromycin resistance cassette, and a 796-bp $5^{\prime}$ part of the gene (Figure 1A). The hygromycin cassette was released from pGEMThyg with SmaI, while the 5'- and 3'-parts of CTB2 were amplified from genomic DNA with primers CTB2-1F, CTB2$2 R$, CTB2-3F, and CTB2-4R. In the fusion PCR, equal amounts of the upper fragment, hygromycin cassette, and lower fragment were fused, and the resulting $3.7-\mathrm{kb}$ fragment was amplified with primers CTB2-nestF and CTB2nestR. The final PCR product was cloned into pGEMT and released with NotI and ApaI.

\section{Fungal transformation}

Protoplasts were prepared as described previously [22], and polyethylene glycol-mediated transformation was carried out as previously described [23]. Buffers were prepared as described previously [24]. Selection with the antibiotic hygromycin B (Duchefa, Harlem, Netherlands) was carried out with $50 \mu \mathrm{g} / \mathrm{ml}$. Retransformation of hygromycin resistant transformants was performed using geneticin (Duchefa, Harlem, Netherlands) selection at a final concentration of $100 \mu \mathrm{g} / \mathrm{ml}$.

\section{Southern blotting}

Genomic DNA from wild type and gene-disruption strains was digested overnight with $B s p H I$ (New England Biolabs, Frankfurt am Main, Germany). The digested DNA was separated on a $0.8 \%$ agarose gel at $80-100 \mathrm{~V}$. DNA was transferred by capillary blotting to a Hybond NX membrane (Amersham Biosciences, Little Chalfont, UK) and then hybridized with a digoxygenin-labeled (Roche, Mannheim, Germany) DNA hygromycin probe. Detection and visualization procedures were followed according to the manufacturer's manual (Roche).

\section{Toxin extraction}

Cercosporin was extracted with $5 \mathrm{~N} \mathrm{KOH}$ as described by Chung [25]. Plates were prepared from PDA at $\mathrm{pH}$ 5.6, evenly inoculated with $C$. beticola conidia, and maintained under daylight conditions and room temperature for two weeks. The agar was cut into small squares and covered with $\mathrm{KOH}$ overnight in a glass beaker. Agar pieces were removed with a small household sieve. The cercosporin contents of the $\triangle C T B 2$ strains of Ahlburg and Ferrara, as well as the wild type strains and the ectopic transformants were measured after extracting toxin from PDA plates cultured for two weeks in ambient light. Each measurement was repeated three times from three independent extractions. A $5 \mathrm{mM}$ cercosporin standard was kindly obtained from Planta GmbH. Absorbance was measured in a spectrometer (Ultrospec 3000, Pharmacia Biotech) at $480 \mathrm{~nm}$ and the cercosporin content was calculated from the molecular weight of $534.51 \mathrm{~g} / \mathrm{mol}$ (Sigma). For testing on plants, cercosporin was extracted from PDA plates directly with water, which worked as well as extraction with $\mathrm{KOH}$. Plants were dipped in plate extract containing approximately $750 \mu \mathrm{M}$ cercosporin (isolate Ahlburg) and $320 \mu \mathrm{M}$ (isolate Ferrara).

\section{Plant inoculation}

Sugar beet plants were grown in a growth chamber at $18^{\circ} \mathrm{C}$ with 16 hours of light. To produce conidia for the plant inoculation, PDA plates ( $\mathrm{pH}$ 5.6) were equally seeded with mycelium mashed in a Waring blender and incubated under daylight for two weeks. Roughly one plate was inoculated for each plant to be infected. The conidia were then carefully scraped off the surface with sterile water and a spatula. The scraped material was filtered through a small household sieve, one layer of miracloth, and a $200 \mu \mathrm{m}$ Wilson sieve to completely remove all agar plucks. The conidia were then counted in a Fuchs-Rosenthal hemocytometer. Twelve-week-old sugar beet plants were inoculated by thoroughly applying $50 \mathrm{ml}$ of a conidia suspension of $2.0^{*} 10^{4}$ conidia/ml onto the adaxial and abaxial leaf surfaces with a spray bottle. Using $50 \mathrm{ml}$ the leaves of one plant were sufficiently covered. Twenty-five plants were inoculated with each fungal strain. The plants were covered with a foil tent for ten days and exposed to 18 hours of light $\left(2.0^{*} 10^{4}\right.$ lux, $\left.400-600 \mathrm{~nm}=4.0-6.0^{*} 10^{3} \mathrm{~K}\right)$ at temperatures of $24^{\circ} \mathrm{C}$ for day and $18^{\circ} \mathrm{C}$ for night.

For testing the toxin on plants grown in vitro, oneweek-old in vitro-cultured plants were dipped into cellfree plate extract or the water control.

\section{Microscopy}

Leaves inoculated with the wild type and $\Delta c t b 2$ strains and strains expressing DsRed were investigated with MZ FL III microscope (Leica Microsystems, Heerbrugg, Switzerland). The microscope was equipped with a Leica 
$1.0 \times$ objective and Leica DFC 500 fluorescence camera. To visualize plant necroses under white light conditions reflected light of an external halogen lamp KL 1500 Electronic (Schott, Mainz, Germany) was used. The DsRed fluorescence was detected with the Leica DsRed excitation filter at 546/12 nm and a long pass filter at $560 \mathrm{~nm}$. The LAS Leica software (version 2.7.1) was used for image acquisition and procession.

High resolution fluorescence microscopy was performed with Zeiss Axio Imager.Z1 microscope equipped with a Zeiss Apotome and AxioCamMRm CCD camera. A UV (ultra violet) lamp HAL 100 served as light source. DsRed was excited in the range of 538 to $562 \mathrm{~nm}$ and detected in the 570 to $640 \mathrm{~nm}$ range. The plant apoplast was excited in the range of 335 to $383 \mathrm{~nm}$ and its blue autofluorescence was detected at 420 to $470 \mathrm{~nm}$. Image processing, including overlay of independently detected DsRed and plant autofluorescence as well as generation of maximum intensity projections (MIP) of $\mathrm{z}$-stacks were done with Zeiss AxioVision software (version 4.8.1). All presented images are MIPs of the respective $\mathrm{z}$-stack.

\section{Disease rating}

Disease ratings were performed according to a method that relates the amount of leaf spots with disease severity [10]. Single leaves were rated according to the following disease index: absence of necrotic areas (leaf spots) $=0$, necrotic area $<1 \%=1$, necrotic area $2-5 \%=2$, necrotic area $6-10 \%=3$, necrotic area $11-20 \%=4$, necrotic area $21-40 \%=5$, necrotic area $41-60 \%=6$, necrotic area $61-80 \%=7$, necrotic area $81-100 \%=8$, and leaf dead $=9$.

\section{Competing Interests}

The authors declare that they have no competing interests.

\section{Authors' contributions}

CS carried out carried out the molecular genetic studies and analytical assays as well as drafted the manuscript. MJB participated in histology and microscopy as well as in drafting the manuscript. CK carried out the fungal transformation. JB, DS and WS coordinated the experimental work, were involved in analysis and interpretation of data as well as in drafting and revising of the manuscript. All authors read and approved the final manuscript.

\section{Author details \\ 'Department of Molecular Phytopathology and Genetics, University of Hamburg, Biocenter Klein Flottbek, Ohnhorststr. 18, Hamburg 22609, Germany. ${ }^{2}$ KWS SAAT AG, Grimsehlstr. 31, Einbeck 37555, Germany.}

Received: 11 July 2012 Accepted: 12 March 2013

Published: 22 March 2013

\section{References}

1. Weltmeier F, Mäser A, Menze A, Hennig S, Schad M, Breuer F, Schulz B, Holtschulte B, Nehls R, Stahl DJ: Transcript profiles in sugar beet genotypes uncover timing and strength of defense reactions to Cercospora beticola infection. Mol Plant Microbe Interact 2011, 24(7):758-772.
2. Weiland J, Koch G: Sugarbeet leaf spot disease (Cercospora beticola Sacc.). Mol Plant Pathol 2004, 5(3):157-166.

3. Rathaiah Y: Stomatal tropism of Cercospora beticola in sugarbeet. Phytopathology 1977, 67:358-362.

4. Feindt $F$, Mendgen $K$, Heitefuss R: Feinstruktur unterschiedlicher Zellwandreaktionen im Blattparenchyn anfälliger und resistenter Rüben (Beta vulgaris L.) nach Infektion durch Cercospora beticola Sacc. Phytopathologische Zeitschrift 1981, 101:248-264

5. Steinkamp MP, Martin SS, Hoefert LL, Ruppel EG: Ultrastructure of lesions produced by Cercospora beticola in leaves of Beta vulgaris. Physiol Plant Pathol 1979, 15(1):13-16. IN13-IN19, 17-26.

6. Daub ME, Ehrenshaft M: The photoactivated Cercospora toxin cercosporin: contributions to plant disease and fundamental biology. Annu Rev Phytopathol 2000, 38:461-490.

7. Chen HQ, Lee MH, Daub ME, Chung KR: Molecular analysis of the cercosporin biosynthetic gene cluster in Cercospora nicotianae. Mol Microbiol 2007, 64(3):755-770

8. Herrero S, Amnuaykanjanasin A, Daub M: Identification of genes differentially expressed in the phytopathogenic fungus Cercospora nicotianae between cercosporin toxin-resistant and -susceptible strains. FEMS Microbiol Lett 2007, 275:326-337.

9. Namiki F, Matsunaga M, Okuda M, Inoue I, Nishi K, Fujita Y, Tsuge T: Mutation of an arginine biosynthesis gene causes reduced pathogenicity in Fusarium oxysporum f. sp melonis. Mol Plant Microbe Interact 2001, 14(4):580-584.

10. Rossi V, Battilani P: Assessment of intensity of Cercospora disease on sugarbeet. Phytopathology 1989, 124(1-4):63-66.

11. Schmidt K, Heberle B, Kurrasch J, Nehls R, Stahl DJ: Suppression of phenylalanine ammonia lyase expression in sugar beet by the fungal pathogen Cercospora beticola is mediated at the core promoter of the gene. Plant Mol Biol 2004, 55(6):835-852.

12. Chen $H Q$, Lee $M H$, Chung KR: Functional characterization of three genes encoding putative oxidoreductases required for cercosporin toxin blosynthesis in the fungus Cercospora nicotianae. Society for General Microbiology 2007, 153:2781-2790.

13. Wiemann P, Willmann A, Straeten M, Kleigrewe K, Beyer M, Humpf H-U, Tudzynski B: Biosynthesis of the red pigment bikaverin in Fusarium fujikuroi: genes, their function and regulation. Mol Microbiol 2009, 72(4):931-946.

14. Studt L, Wiemann P, Kleigrewe K, Humpf H-U, Tudzynski B: Biosynthesis of fusarubins accounts for pigmentation of Fusarium fujikuroi perithecia. Appl Environ Microbiol 2012, 78(12):4468-4480.

15. Choquer M, Dekkers KL, Chen H-Q, Cao L, Ueng PP, Daub ME, Chung K-R: The CTB1 gene encoding a fungal polyketide synthase is required for cercosporin biosynthesis and fungal virulence of Cercospora nicotianae. Mol Plant Microbe Interact 2005, 18(5):468-476.

16. Weiland JJ, Chung KR, Suttle JC: The role of cercosporin in the virulence of Cercospora spp. to plant hosts. In Cercospora leaf spot of sugar beet and related species. Edited by Lartey RT, Weiland JJ, Panella L, Crous PW, Windels CE. St. Paul, USA: The American Phytopathological Society; 2010.

17. Steinkamp MP, Martin SS, Hoefert LL, Ruppel EG: Ultrastructure of lesions produced in leaves of Beta vulgaris by cercosporin, a toxin from Cercospora beticola. Phytopathology 1981, 71(12):1272-1281.

18. Schmidt K, Pflugmacher M, Klages S, Maser A, Mock A, Stahl DJ: Accumulation of the hormone abscisic acid (ABA) at the infection site of the fungus Cercospora beticola supports the role of ABA as a repressor of plant defence in sugar beet. Mol Plant Pathol 2008, 9(5):661-673.

19. Ehrenshaft M, Upchurch RG: Isolation of light-enhanced cDNAs of Cercospora kikuchii. Appl Environ Microbiol 1991, 57:2671-2676.

20. Harris S: Morphogenesis in germinating Fusarium graminearum macroconidia. Mycologia 2005, 97(4):880-887.

21. Lindsey K, Gallois P: Transformation of sugarbeet (Beta vulgaris) by Agrobacterium tumefaciens. Experimental Botany 1990, 41(5):529-536.

22. Jenczmionka NJ, Maier FJ, Lösch AP, Schäfer W: Mating, conidiation and pathogenicity of Fusarium graminearum, the main causal agent of the head-blight disease of wheat, are regulated by the MAP kinase gpmk1. Curr Genet 2003, 43(2):87-95.

23. Proctor R, Hohn TM, McCormick SP: Reduced virulence of Gibberella zeae caused by disruption of a trichothecene toxin biosynthetic gene. Mol Plant Microbe Interact 1995, 8(4):593-601. 
24. Chung KR, Ehrenshaft M, Daub ME: Functional expression and cellular localization of cercosporin-resistance proteins fused with the GFP in Cercospora nicotianae. Curr Genet 2002, 41(3):159-167.

25. Chung KR: Involvement of calcium/calmodulin signaling in cercosporin toxin biosynthesis by Cercospora nicotianae. Appl Environ Microbiol 2003, 69(2):1187-1196.

doi:10.1186/1471-2229-13-50

Cite this article as: Staerkel et al: СbCTB2, an O-methyltransferase is essential for biosynthesis of the phytotoxin cercosporin and infection of sugar beet by Cercospora beticola. BMC Plant Biology 2013 13:50.

\section{Submit your next manuscript to BioMed Central and take full advantage of:}

- Convenient online submission

- Thorough peer review

- No space constraints or color figure charges

- Immediate publication on acceptance

- Inclusion in PubMed, CAS, Scopus and Google Scholar

- Research which is freely available for redistribution 